PROCEEDINGS OF THE

AMERICAN MATHEMATICAL SOCIETY

Volume 140, Number 12, December 2012, Pages 4243-4245

S 0002-9939(2012)11281-X

Article electronically published on April 17, 2012

\title{
A TREE CHARACTERIZATION OF THE POINT OF CONTINUITY PROPERTY IN GENERAL BANACH SPACES
}

\author{
GINÉS LÓPEZ PÉREZ AND JOSÉ ANTONIO SOLER ARIAS
}

(Communicated by Thomas Schlumprecht)

\begin{abstract}
We obtain a characterization of the point of continuity property for general bounded subsets in Banach spaces in terms of trees. For this we introduce the notion of a topologically weakly null tree and, as a consequence, we get that a general Banach space satisfies the point of continuity property if, and only if, every seminormalized topologically weakly null tree has a boundedly complete branch.
\end{abstract}

\section{INTRODUCTION}

A bounded subset $K$ of a Banach space has the point of continuity property (PCP) if every nonempty closed subset admits a point of continuity for the identity map from the weak to norm topologies. From [1] it is known that $K$ fails PCP iff there exists a countable set $C \subset K$ and $\delta>0$ so that diam $(U)>\delta$ for all nonempty relatively open $U \subset C$.

A Banach space $X$ has the PCP if its closed unit ball has the PCP. Furthermore, from [3] and [5], a separable $X$ has the PCP iff it admits a boundedly complete skipped blocking finite dimensional decomposition (BCSBFDD) $\left(F_{j}\right)_{j=1}^{\infty}$. This means that $X=\left[F_{j}: j \in \mathbb{N}\right], F_{j} \cap\left[F_{k}: k \neq j\right]=\{0\}$ for all $j$ and if $\left(x_{n}\right)$ is any seminormalized skipped block sequence w.r.t. $\left(F_{j}\right)$, then $\left(x_{j}\right)$ is a boundedly complete basic sequence. $\left(x_{j}\right)$ is skipped w.r.t. $\left(F_{j}\right)$ means that for some $p_{1}<p_{2}<\ldots$, $x_{j} \in\left[F_{i}: p_{j}<i<p_{j+1}\right]$. For $S \subseteq X,[S]$ denotes the closed linear span of $S$.

Much has been written about the PCP, and we refer the reader to [1], 3] and [5] for further background.

In this paper we prove

Theorem A. Let $K$ be a bounded subset of a Banach space $X$. The following are equivalent:

1) $K$ fails the PCP.

2) There exists a seminormalized topologically weakly null tree $\left\{x_{A}\right\}_{A \in[\mathbb{N}<\omega]}$ in $X$ such that

$$
\left\{\sum_{B \leq A} x_{B}: A \in[\mathbb{N}]^{<\omega}\right\} \subseteq K
$$

Received by the editors February 18, 2011 and, in revised form, March 9, 2011; April 11, 2011; May 23, 2011; and May 30, 2011.

2010 Mathematics Subject Classification. Primary 46B20, 46B22.

Key words and phrases. Point of continuity property, trees, boundedly complete sequences.

This work was partially supported by MEC (Spain) Grant MTM2006-04837 and Junta de Andalucía Grants FQM-185 and Proyecto de Excelencia P06-FQM-01438. 
Theorem B. Let $X$ be a Banach space. The following are equivalent:

i) $X$ has the PCP.

ii) Every seminormalized topologically weakly null tree in $X$ admits a boundedly complete branch.

$[\mathbb{N}]^{<\omega}$ denotes the set of all finite sequences in $\mathbb{N}$ ordered by extension. A branch of a tree $\left\{x_{A}\right\}$ is a sequence $\left(x_{\emptyset}, x_{\left(n_{1}\right)}, x_{\left(n_{1}, n_{2}\right)}, \ldots\right)$ for some sequence $\left(n_{i}\right)_{1}^{\infty}$ of positive integers. A tree $\left\{x_{A}\right\}$ is weakly null if $x_{A, n} \stackrel{\omega}{\rightarrow} 0$ as $n \rightarrow \infty$ for all $A \in[\mathbb{N}]^{<\omega}$. It is topologically weakly null if $0 \in{\overline{\left\{x_{A, n}\right\}_{n=1}^{\infty}}}^{\omega}$.

Theorem $\mathrm{B}$ was known in the case that $X^{*}$ is separable 4 or more generally if $X$ contains no isomorph of $\ell_{1}[6$, where in ii) the word "topologically" is deleted. If $X$ does not contain $\ell_{1}$, then any sequence with 0 in its weak closure contains a subsequence that is weakly null (see [2] and [8]). It follows that any topologically weakly null tree in a space not containing $\ell_{1}$ admits a full subtree that is weakly null. Hence Theorem B yields these earlier results and extends them to a general characterization.

\section{THE PROOFS}

Proof of Theorem A. 1) $\Rightarrow \mathbf{2}$ ). If $K$ fails the PCP there exist $\delta>0$ and a countable set $C \subseteq K$ with diam $U>2 \delta$ for all relatively open nonempty $U \subseteq C$. Let $y_{\emptyset} \in C$. Then $y_{\emptyset} \in \overline{C \backslash B\left(y_{\emptyset}, \delta\right)^{\omega}}$, where $B\left(y_{\emptyset}, \delta\right)$ is the closed $\delta$-ball centered at $y_{\emptyset}$. Order $C \backslash B\left(y_{\emptyset}, \delta\right)$ as $\left(y_{(n)}\right)_{n=1}^{\infty}$. Note that $y_{\emptyset} \in{\overline{\left\{y_{(n)}\right.}}^{\omega}$. We then repeat this step for each $y_{(n)}$ to obtain $y_{(n, m)}$ 's and so on for each $y_{A}$ once it is defined.

Set $x_{\emptyset}=y_{\emptyset}$ and for $A \in[\mathbb{N}]^{<\omega}$, set $x_{(A, n)}=y_{(A, n)}-y_{A}$. It follows that $\left\|x_{A}\right\|>\delta$ for all $A$ (we could have chosen $\left\|y_{\emptyset}\right\|>\delta$ to start) and $\left\{x_{A}\right\}$ is a topologically weakly null tree. Also for all $A \in[\mathbb{N}]^{<\omega}$,

$$
\sum_{B \leq A} x_{B}=y_{A} \in K
$$

2) $\Rightarrow$ 1). Let $C=\left\{\sum_{B<A} x_{B}: A \in[\mathbb{N}]^{<\omega}\right\}$. Then $C$ satisfies the criterion mentioned earlier for failing the PCP.

We next prove Theorem B. First we add two equivalent conditions:

iii) Every seminormalized topologically weakly null tree $\left\{x_{A}\right\}$ in $X$ satisfies

$$
\sup \left\{\sum_{j=1}^{k} x_{\left(n_{1}, \ldots, n_{j}\right)}: k \in \mathbb{N}, n_{1}, n_{2}, \ldots \in \mathbb{N}\right\}=\infty .
$$

iv) For every seminormalized topologically weakly null tree $\left\{x_{A}\right\}$ in $X$ there exist integers $n_{1}, n_{2}, \ldots \in \mathbb{N}$ with

$$
\sup _{k}\left\|\sum_{j=1}^{k} x_{\left(n_{1}, \ldots, n_{j}\right)}\right\|=\infty .
$$

Proof of Theorem B. ii $\Rightarrow$ iv $) \Rightarrow$ iii) are trivial. iii) $\Rightarrow$ i) follows from Theorem A. i) $\Rightarrow$ ii) follows the fact that $\left[x_{A}: A \in[\mathbb{N}]^{<\omega}\right]$ is a separable space with the PCP and thus has a BCSBFDD $\left(F_{j}\right)$. Indeed it is easy to see that if $\left\{x_{A}\right\}$ is a seminormalized topologically weakly null tree, then some branch is a perturbation of a skipped block sequence w.r.t. $\left(F_{j}\right)$ and hence is boundedly complete. 
Remarks. 1. It seems unlikely that given a seminormalized topologically weakly null tree one can select a full subtree which is topologically weakly null and basic. This is easily done if the original tree is weakly null. It remains open if $X$ fails the PCP implies $X$ contains a subspace $Y$, failing the PCP, with a basis.

2. Tree characterizations are often equivalent to certain games. In the infinite asymptotic game played in a separable space $X$, (I) chooses $X_{1}$ a finite co-dimensional subspace of $X$ and (II) chooses $x_{1}$ in the unit sphere of $X_{1}$. Plays alternate thusly forever.

Problem. Is $X$ having the PCP equivalent to (I) having a winning strategy to force (II) to select a boundedly complete basic sequence?

This is the case if $X^{*}$ is separable (see [7).

\section{ACKNOWLEDGMENT}

The authors thank the referee for helping them to rewrite this paper.

\section{REFERENCES}

[1] J. Bourgain, Dentability and finite-dimensional decompositions. Studia Math. 67 (1980), 135148. MR.583294 (81m:46034)

[2] J. Bourgain, D. H. Fremlin, M. Talagrand, Pointwise compact sets of Baire-measurable functions. Amer. J. Math. 100 (1978) (4), 845-886. MR509077 (80b:54017)

[3] J. Bourgain, H. P. Rosenthal, Geometrical implications of certain finite dimensional decompositions. Bull. Belg. Math. Soc. Simon Steven 32 (1980), 54-75. MR682992 (84d:46022)

[4] S. Dutta, V. P. Fonf. On tree characterizations of $G_{\delta}$-embeddings and some Banach spaces. Israel J. Math. 167 (2008), 27-48. MR2448016(2009i:46024)

[5] N. Ghoussoub, B. Maurey. $G_{\delta}-$ embeddings in Hilbert spaces. J. Funct. Anal. 61 (1985), 72-97. MR.779739 (86m:46016)

[6] G. López-Pérez, J. A. Soler-Arias, The convex point of continuity property in Banach spaces not containing $\ell_{1}$. J. Math. Anal. Appl. 378 (2011), 734-740. MR2773281

[7] E. Odell, T. Schlumprecht, Trees and branches in Banach spaces. Trans. Amer. Math. Soc. 354 (10) (2002), 4085-4108. MR1926866 (2003h:46015)

[8] H. P. Rosenthal, Point-wise compact subsets of the first Baire class. Amer. J. Math. 99 (2) (1977), 362-378. MR0438113(55:11032)

Departamento de Análisis Matemático, Facultad de Ciencias, Universidad de GraNADA, 18071-GranadA, SPAIN

E-mail address: glopezp@ugr.es

Departamento de Análisis Matemático, Facultad de Ciencias, Universidad de GraNADA, 18071-GRANADA, SPAIN

E-mail address: jasoler@ugr.es 